PROCEEDINGS OF THE

AMERICAN MATHEMATICAL SOCIETY

Volume 133, Number 11, Pages 3317-3321

S 0002-9939(05)07889-5

Article electronically published on May 9, 2005

\title{
A NEW REPRESENTATION OF THE DEDEKIND COMPLETION OF $C(K)$-SPACES
}

\author{
Z. ERCAN AND S. ONAL
}

(Communicated by Joseph A. Ball)

\begin{abstract}
A new representation of the Dedekind completion of $C(K)$ is given. We present a necessary and sufficient condition on a compact Hausdorff space $K$ for which the Dedekind completion of $C(K)$ is $B(S)$, the space of real valued bounded functions on some set $S$.
\end{abstract}

\section{INTRODUCTION}

As usual for a topological space $K$, the space of real valued continuous (bounded) functions on $K$ is denoted by $C(K)\left(C_{b}(K)\right)$. The characteristic function of $k \in K$ is denoted by $\chi_{k}$. For a given set $S, B(S)$ denotes the space of real valued bounded functions on $S$. We refer to [6] for classical terminology on Riesz space theory.

Recall that a Dedekind complete Riesz space $F$ is called a Dedekind completion of a Riesz space $E$ if there exists a Riesz isomorphism $\pi$ from $E$ into $F$ such that for each $0<x \in F$ there exist $0<a, b \in E$ such that

$$
\pi(a) \leq x \leq \pi(b) .
$$

It is well known that each Archimedean Riesz space has a unique (up to Riesz isomorphism) Dedekind completion. For a compact Hausdorff space $K, C(K)$ is Dedekind complete if and only if $K$ is Stonean. This is due to Nakano [9]. Recall that a compact Hausdorff space is called Stonean if the closure of each open set is also open. In the literature there are several descriptions of the Dedekind completion of the $C(K)$-spaces. Some of these are:

- Let $K$ be a compact Hausdorff space. Dilworth [2] has given a description of the Dedekind completion of $C(K)$ in terms of lower and upper semicontinuous functions.

- Nakano-Shimagaki [10] showed that the Dedekind completion of $C(K)$ can be constructed as a semi-ordered linear space.

- In [7 Mack and Johnson have given a necessary and sufficient condition that the Dedekind completion of $C(X)$, for $X$ a realcompact space, be isomorphic to $C(Y)$ for some $Y$. This is obtained for the class of countably paracompact and normal spaces in [12] by Weinberg.

Received by the editors November 30, 2003 and, in revised form, June 21, 2004.

2000 Mathematics Subject Classification. Primary 46A40; Secondary 46B42, 54B42.

Key words and phrases. Riesz spaces, Dedekind completion.

(C)2005 American Mathematical Society Reverts to public domain 28 years from publication 
- Maxey 8 ] has shown that for any maximal ideal $I$ of $C(K)^{\sim \sim}$ with $C(K) \cap I=$ $\{0\}$, the quotient space $C(K)^{\sim \sim} / I$ is the Dedekind completion of $C(K)$. This is Theorem 1.49 of [1].

- In [13 and [14] Zaharov has given the Dedekind completion of $C(K)$ in terms of quasi normal functions and functions with Baire property. These results are generalized in [11] by Veksler.

- In 44 and [5] Kaplan has presented several descriptions of the Dedekind completion of $C(K)$.

We also refer to [3] for some more details about these results.

It is surprise that, to the best of our knowledge, there has been no investigation on the following question, which was asked by the first author in the 2003 Positivity Conference in Rhodes:

For what type of compact Hausdorff space K, the Dedekind completion of $C(K)$ is $B(S)$ for some set $S$ ?

The aim of this paper is to give a new representation of the Dedekind completion of $C(K)$-space and then answer the above question.

\section{MAin Results}

We begin with the following definition.

Definition 1. A surjective map $\varphi$ from a completely regular Hausdorff space $X$ into another one $Y$ is called irreducible if $\varphi(A) \neq Y$ for each closed proper subset $A$ of $X$.

Let $X$ and $Y$ be completely regular Hausdorff spaces and let $\varphi$ be a continuous closed surjective function from $X$ into $Y$. Then we define

$$
\varphi^{*}: C_{b}(Y) \longrightarrow C_{b}(X), \quad \varphi^{*}(f)=f \varphi .
$$

It is clear that $\varphi^{*}$ is an isometry and Riesz isomorphism from $C_{b}(Y)$ into $C_{b}(X)$.

Lemma 2. Let $X$ and $Y$ be completely regular Hausdorff spaces and let $\varphi$ be a continuous closed surjection from $X$ to $Y$. Then $\varphi^{*}\left(C_{b}(Y)\right)$ is order dense in $C_{b}(X)$ if and only if $\varphi$ is irreducible.

Proof. Suppose that $\varphi^{*}(C(Y))$ is order dense in $C(X)$ and $\varphi$ is not irreducible. Choose a closed subset $A$ of $X$ with $\varphi(A)=Y$. Let $p \in X \backslash A, 0 \leq f \in C(X)$ with $f(p)=1$ and $f(A)=\{0\}$. Then there exists $g \in C(Y)$ such that $0<\varphi^{*}(g) \leq f$. As $\varphi(A)=Y$ we have $g=0$, which is a contradiction.

Conversely, suppose that $\varphi$ is irreducible. Let $0<f \in C(X)$. Choose $0<r \in \mathbf{R}$ and a nonempty open set $U$ in $X$ such that $f(U) \subset(r, \infty)$. As $X \backslash U$ is a closed proper subset of $X, \varphi(X \backslash U)$ is a proper closed subset of $Y$. We can have $q \in$ $Y \backslash \varphi(X \backslash U), g \in C(Y)$ such that

$$
g(q)=1 \quad \text { and } \quad g(\varphi(X \backslash U))=\{0\} .
$$

Then we have $\varphi \subset[0, r]$ and $0<\varphi^{*}(g) \leq f$. This completes the proof.

Let $K$ be a topological space and let $A \subset K$. Then $\operatorname{cl}(A)$ denotes the closure of $A$. Similarly $\operatorname{Int}(A)$ stands for the interior of $A$. A lemma similar to the following may be known, and its proof is routine. We prove only its last part. 
Lemma 3. Let $X$ be a compact Hausdorff space. Then

i.

$$
R O(X)=\{O \subset X: O=\operatorname{int}(\operatorname{cl}(O))\}
$$

is a Dedekind complete Boolean algebra under the operations

$$
U \vee V=\operatorname{Int}(\operatorname{cl}(U \cup V)), \quad U \wedge V=U \cap V
$$

with $e=X$ and $0=\emptyset$.

ii. Let $Y=$ Stone $R O(X)$ be the set of all maximal filters in $R O(X)$. Then $Y$ is a Stonean space with the basis

$$
\left\{U^{*}=\{p: U \in p\}: U \in R O(X) \backslash\{\emptyset\}\right\} .
$$

iii. For each $p \in Y$, there exits $x_{p} \in X$ with $\bigcap_{U \in p} c l(U)=\left\{x_{p}\right\}$ and

$$
\varphi: Y \longrightarrow X, \quad \varphi(p)=x_{p}
$$

is continuous, surjective and irreducible.

Proof. We prove only that $\varphi$ is irreducible (the proof of the other parts of the lemma is clear). Let $F$ be a proper closed subspace $Y$ and let $q \in Y \backslash F$. Choose $U \in R O(X)$ such that

$$
U^{*} \cap F=\emptyset, \quad q \in U .
$$

Let $p \in F$. Then $p \notin U^{*}$, so $U \notin p$. As $p$ is maximal, $p \cup\{U\}$ is not a filter. Then there exits $V \in p$ with $V \cap U=\emptyset$. Then

$$
\bigcap_{O \in p} \operatorname{cl}(O) \subset \operatorname{cl}(V) \text { and } \quad U \cap \operatorname{cl}(V)=\emptyset .
$$

Thus, $\varphi(p) \notin U$ for each $p \in F$. Hence $\varphi(F) \neq X$. This completes the proof.

Now we are ready to prove one of the main theorems.

Theorem 4. Let $X$ be a compact Hausdorff space and let $Y=\operatorname{Stone} R O(X)$. Then the Dedekind completion of $C(X)$ is $C(Y)$.

Proof. Consider the isometry and order isomorphism

$$
\varphi^{*}: C(X) \longrightarrow C(Y), \quad \varphi^{*}(f)=f \varphi,
$$

where $\varphi$ is defined as in the above lemma (iii). Since $\varphi$ is continuous surjective and irreducible from Lemma $2, \varphi^{*}(C(X))$ is an order dense Riesz subspace of $C(Y)$ and dominates the elements of $C(Y)$. Since $Y$ is Stonean, $C(Y)$ is also Dededekind complete. This completes the proof.

It is well known that each AM-space $E$ with a strong order unit can be represented by $C\left(K_{E}\right)$ for some compact Hausdorff space $K_{E}$ as Riesz isomorphic spaces. $K_{E}$ is known as the Kakutani-Krein space of $E$. The corollary below follows immediately from the above theorem.

Corollary 5. Let $E$ be an AM-space with a strong unit. Then $C(Y)$ is the Dedekind completion of $E$, where $Y=S$ Stone $R O\left(K_{E}\right)$ and $K_{E}$ is the Kakutani-Krein space of $E$.

Recall that a nonzero positive element $x$ in an Archimedean Riesz space is called an atom if the (order) ideal generated by $x$ is one dimensional. The following lemma is well known and the proof is trivial, so we omit its proof. 
Lemma 6. Let $K$ be a compact Hausdorff space. Then $0<f \in C(K)$ is an atom if and only if $f=\alpha \chi_{a}$ for some isolated point $a \in X$ and $0 \leq \alpha \in \mathbf{R}$.

Now we can give an answer to the question which was asked in the Introduction.

Theorem 7. Let $K$ be a compact Hausdorff space. Then the Dedekind completion of $C(K)$ is $B(S)$ for some $S$, if and only if the set of isolated points of $K$ is dense in $K$.

Proof. Suppose that the Dedekind completion of $C(K)$ is $B(S)$, and let $\pi$ be the required isomorphism from $C(K)$ into $B(S)$. Let $A$ be the set of isolated points of $S$. Let $s \in S$ be given. Then there exists $0<f \in C(K)$ such that $\pi(f) \leq \chi_{s}$, so $\pi(f)=\alpha \chi_{s}$ for some positive real number $\alpha$. As $\pi^{-1}\left(\chi_{s}\right)$ is an atom, $f$ is an atom, so there exists a unique isolated point $\sigma(s) \in K$ such that $f=\beta \chi_{\sigma(s)}$ for some real number $\beta$. That is, we have a one-to-one map $\sigma$ from $S$ into $A$. Let $a \in A$ be given. Then $\pi\left(\chi_{a}\right)=\alpha \chi_{s}$ for some $s \in S$ and real number $\alpha$. This observation shows that $a=\sigma(s)$, so $\sigma$ is also onto. So, $S$ can be replaced by $A$. Suppose that $A$ is not dense in $K$. Then there exists $k \in K$ and $f \in C(K)$ such that $f(k)=1$ and $f=0$ on $A$. As $0<\pi(f)$ there exists $a \in A$ with $0<\alpha \chi_{a} \leq \pi(f)$ for some $\alpha$. Then $\pi^{-1}\left(\chi_{a}\right)$ is zero on $A$. Since it is an atom, there exists an isolated point $k \in A$ with $\pi^{-1}\left(\chi_{a}\right)=\alpha \chi_{k}$ for some nonzero positive real number $\alpha$. This is a contradiction, so $A$ must be dense in $K$.

Now suppose that $A$ is dense in $K$. Define $\pi: C(K) \longrightarrow B(A)$ by $\pi(f)=\left.f\right|_{A}$. Let $0<f \in B(A)$. Choose $a \in A$ with $0<f(a) \chi_{a} \leq f$. Then

$$
0<\pi\left(f(a) \chi_{a}\right) \leq f \leq \pi(\|f\| \mathbf{1}) .
$$

This completes the proof.

We can give an alternative proof of the above theorem as follows: Let $\pi$ : $C(X) \longrightarrow B(S)$ be an order dense Riesz isomorphism with $\pi(\mathbf{1})=\mathbf{1}$. Then there exists $\varphi: \beta S \longrightarrow X$ which is a continuous surjection such that $\varphi^{*}=\pi$. Then from Lemma 2, $\varphi$ is irreducible and $\operatorname{cl}(\varphi(S))=X$ (from the fact that $X=\varphi(\beta S) \subset \operatorname{cl}(\varphi(S)))$. For each $q \in S,\{q\}$ is open in $\beta S$ and $\beta S \backslash\{q\}$ is a proper closed subset of $X$. Then $\varphi(\beta S \backslash\{q\})$ is a proper closed subset of $X$. As $X \backslash \varphi(\beta S \backslash\{q\})$ is open and

$$
\varphi(q)=X \backslash \varphi(\beta S \backslash\{q\}),
$$

$\varphi(q)$ is an isolated point in $X$ and $\{\varphi(q): q \in S\}\}$ is dense in $X$, so the set of isolated points in $X$ is dense in $X$.

The following corollary immediately follows from the above theorem.

Corollary 8. Let $K$ be a Stonean space. The set of isolated points is dense in $K$ if and only if $K$ is a Stone-Cech compactification of a discrete space $S$.

Let $K$ be a discrete topological space. Then from the above theorem the Dedekind completion of $C\left(K_{\infty}\right)$ is $B(K)$, where $K_{\infty}$ is a one-point compactification of $K$. Each compact Hausdorff space $K$ generates a compact Hausdorff space $A(K)$ such that $K$ is homeomorphic into $A(K)$ and the set of isolated points is dense in $A(K)$. This construction is called the Alexandroff Duplicate of $K$, that is, $A(K)$ is a compact Hausdorff space on $K \times\{0,1\}$ generated by sets of the form $F \times\{1\}$ and $U \times\{0,1\} \backslash G \times\{1\}$, where $F, G \subset K$ with $G$ is finite and $U$ is open 
in $K$. Then for each $k \in K,(k, 1)$ is an isolated point and $K \times\{1\}$ is dense in $A(K)$. Hence the Dedekind completion of $C(A(K))$ is $B(K \times\{1\})(=B(K))$. This provides many examples of compact Hausdorff spaces $K$ such that the Dedekind completion of $C(K)$ is $B(S)$.

\section{REFERENCES}

[1] C. D. Aliprantis and O. Burkinshaw, Locally Solid Riesz spaces with Applications to Economics, $2^{\text {nd }}$ Edition, Mathematical Surveys and Monographs, Volume 105, American Mathematical Society, Providence, RI, 2003. MR2011364

[2] R. P. Dilworth, The normal completion of the lattice of continuous functions, Trans. Amer. Math. Soc. 68 (1950), 427-438. MR.0034822 (11:647g)

[3] W. Filter, Wolfgang Representations of Archimedean Riesz spaces-a survey, Rocky Mountain J. Math. 24 (1994), no. 3, 771-851. MF1307578 (96f:46006)

[4] S. Kaplan, The Bidual of $C(X)$ I, North-Holland, New York and Amsterdam, 1985. MR0776606 (86k:46001)

[5] S. Kaplan, Lebesgue Theory in the Bidual of $C(X)$, Memoirs Amer. Mat. Society 121 (1996), no. 579. MR 1329941 (96i:46023)

[6] W. A. J. Luxemburg and A.C. Zaanen, Riesz spaces I, North-Holland Publ. Comp., Amsterdam, 1971. MR0511676 (58:23483)

[7] J. E. Mack and D.G. Johnson, The Dedekind completion of $C(X)$, Pacific J. Math. 20 (1967), 231-243. MR0211268 (35:2150)

[8] W. Maxey, The Dedekind completion of $C(X)$ and its second dual, Ph.D. Thesis (1973), Purdue Univ.

[9] N. Nakano, Über das System aller stetigen Funktionen auf einen Topologischen Raum, Proc. Imp. Acad. (Tokyo) 17 (1941), 308-310. MR0014173(7:249f)

[10] N. Nakano and T. Shimogaki, A note on the cut extension of $C$-spaces, Proc. Japan. Acad. 38 (1962), 473-477. MR0149254 (26:6744)

[11] A. I. Veksler, Functional representation of the order complement of a vector lattice of continuous functions, Sibirsk. Mat. Zh. 26 (1985), no. 6, 159-162. MR0816514 (87j:46048a)

[12] E. C. Weinberg, The alpha-completion of ring of continuous real-valued functions, Notices Amer. Math. Soc. 7 (1960), 533-534.

[13] V. K. Zaharov, Functional characterization of absolute and Dedekind completion, Bull. Acad. Polon. Sci. Ser. Math. 29, no.5-6 (1981), 293-297. MR0640475 (83b:54019)

[14] V. K. Zaharov, Functional characterization of the absolute, vector lattices of functions with Baire property and of quasinormal functions and modules of particular continuous functions, Trudy Moskov. Mat. Obshch. 45 (1982), 68-104. MR0704628 (85c:46033)

Department of Mathematics, Middle East Technical University, 06531 Ankara, TURKEY

E-mail address: zercan@metu.edu.tr

Department of Mathematics, Middle East Technical University, 06531 Ankara, TURKEY

E-mail address: osul@metu.edu.tr 